\title{
Using Padding to Optimize Locality in Scientific Applications
}

\author{
E. Herruzo ${ }^{1}$, O. Plata ${ }^{2}$, and E.L. Zapata ${ }^{2}$ \\ ${ }^{1}$ Dept. Electronics, University of Córdoba, Spain \\ eze@uco.es \\ ${ }^{2}$ Dept. Computer Architecture, University of Málaga, Spain \\ oscar@ac.uma.es, ezapata@ac.uma.es
}

\begin{abstract}
Program locality exploitation is a key issue to reduce the execution time of scientific applications, so as many techniques have been designed for locality optimization. This paper presents new compiler algorithms based on array padding that optimize program locality either locally (at loop level) or globally (the whole program). We first introduce a formal cache model that is used to analyze how all cache levels are filled up when arrays inside nested loops are referenced. We further study the relation between the model parameters and the data memory layout of the arrays, and define how to pad those arrays in order to optimize cache occupation at all levels. Experimental evaluation on some numerical benchmarks shows the benefits of our approach.
\end{abstract}

\section{Introduction}

For the last decades locality exploitation has been one of the main goals for improving the performance of scientific applications, giving rise to a wide range of software optimizations. Nowadays two locality-related trends can be observed: applications process larger and larger data sets, and the processor-memory gap problem is more and more significant. The memory latency problem has been attacked from two different fronts. On the one hand, by means of hardware solutions, like lockup-free caches, multithreading, prefetching, out-of-order execution, data and instruction speculation and so on. On the other hand, by means of compiler techniques for code and/or data transformations [1].

Array padding is a well-known data layout optimization technique that optimizes locality by reducing conflict misses. Although a global technique (affects the whole program), its use can be localized to the nested loop (or few loops) where most of the execution time is spent (a frequent case in scientific applications). This paper presents a simple model of the cache that captures essential information of its behaviour during the execution of a loop nest. This model is used as a framework to define how to pad the arrays in the loop in order to optimize cache occupation. Our method establishes a relationship among a small set of cache parameters, how the array elements are referenced and how they are stored in memory in order to obtain the optimal padding that optimizes cache occupation. The proposed method is subsequently extended to optimize cache 
locality for the whole program (global code optimization) and as well as for the complete cache hierarchy.

The rest of the paper is organized as follows. First, the cache model developed to design our padding methods is introduced. In the next section, our intra-array padding approach is developed for three scenarios: a single loop nest optimization, all loops in the program (global optimization) and all levels in the cache hierarchy. After that, the proposed techniques are experimentally evaluated for a wide set of numerical bechmarks. Finally, some related work is discussed.

\section{Modelling the Cache Behaviour}

A minimal set of characteristic parameters will be defined with the aim of optimizing cache occupation by using array padding. We consider a $L$-way setassociative cache, of size $C \times L \times W$, where $C$ is the number of cache sets, $L$ is the number of blocks per set, and $W$ is the block size in words. We also consider that the array access patterns come from referencing a $M$-dimensional array, $X$, within a $N$-depth nested loop. Expressions in the array dimensions are of the form $f_{k} * I_{k}, k=1, \ldots, M$, where $\boldsymbol{I}=\left(I_{1}, I_{2}, \ldots, I_{N}\right)$ is the iteration vector of the loop, but any general affine expression is perfectly valid. Without loss of generality, we assume that $N=M$. To simplify the explanation, we restrict the cache model to a single array $X$ inside a perfectly nested loop. However this model can be extended to several arrays appearing in the same loop and to not-perfectly nested loops.

When the multidimensional array $X$ is allocated in memory, it is linearized and laid out in some order. So, considering for instance a column-major order, the offset (in words) from the beginning of the array of the element of $X$ referenced in the iteration $\boldsymbol{I}=\left(I_{1}, I_{2}, \ldots, I_{M}\right)$ of the nested loop is given by $\operatorname{ArrOff}(X, \boldsymbol{I})=$ $f_{1} * I_{1}+\ldots+f_{k} * I_{k} * \prod_{i=1}^{k-1} D_{i}+\ldots+f_{M} * I_{M} * \prod_{i=1}^{M-1} D_{i}$, where $D_{i}$ is the size of the $i$-dimension of $X$. The stride of array $X$ on loop index $I_{k}$ is defined as the distance in memory (in words) of array entries referenced by consecutive iterations of the loop $k$, that is, $\operatorname{Stride}\left(X, I_{k}\right)=f_{k} * I_{k}^{l+1} * \prod_{i=1}^{k-1} D_{i}-f_{k} * I_{k}^{l} *$ $\prod_{i=1}^{k-1} D_{i}$, where $I_{k}^{l}$ represents the $l$-th iteration of the loop $k$.

Let us consider now the cache. The execution of consecutive iterations of the loop $k$ generates array references separated in memory a word distance equals to $\operatorname{Stride}\left(X, I_{k}\right)$. We will define now two new cache-related strides. The above array references are contained in cache blocks (of size $W$ words each). The distance (in blocks) between these cache blocks is defined as the cache block stride, that is, $\operatorname{BlockStride}\left(X, I_{k}\right)=\operatorname{block}\left(X, I_{k}^{l+1}\right)-\operatorname{block}\left(X, I_{k}^{l}\right)=$ $\left\lfloor\operatorname{Mem} \operatorname{Addr}\left(X, I_{k}^{l+1}\right) / W\right\rfloor-\left\lfloor\operatorname{Mem} \operatorname{Addr}\left(X, I_{k}^{l}\right) / W\right\rfloor$. Note that although Stride() is constant BlockStride() may not be constant, depending on the relative offset of the memory references in their corresponding blocks. On the other hand, the distance (in cache sets) of the above blocks once they are placed into cache is defined as the cache set stride, that is, $\operatorname{Set} \operatorname{Stride}\left(X, I_{k}\right)=\left(\operatorname{block}\left(X, I_{k}^{l+1}\right)-\right.$ $\left.\operatorname{block}\left(X, I_{k}^{l}\right)\right) \bmod C$, or $\operatorname{Set} S t r i d e\left(X, I_{k}\right)=\operatorname{BlockStride}\left(X, I_{k}\right) \bmod C$. We are assuming BlockStride is a positive integer, in order to simplify the expressions 
(otherwise, we have to take absolute values and consider negative loop steps). With this definition, if we take as a reference the first iteration of the loop $k$ (iteration $L w$ ), we can use the following expression to calculate the set where is located the block referenced in the $l$-th iteration of the loop,

$$
\operatorname{Set}\left(X, I_{k}^{l}\right)=\left(\operatorname{Set}\left(X, I_{k}^{L w}\right)+l * \operatorname{Set} \operatorname{Stride}\left(X, I_{k}\right)\right) \bmod C .
$$

This expression assumes that SetStride() is constant for all iterations of the loop $k$, which is true if BlockStride() is also constant for that loop. This condition is fulfilled if all array references have the same block offset, that is, if $\operatorname{Stride}\left(X, I_{k}\right)$ is a multiple of $W$. Our method to optimize cache occupation considers that this is the case. Otherwise, we can always reach this condition by incrementing the $k$ dimension of $X$ by the amount $\operatorname{Stride}\left(X, I_{k}\right) \bmod W$.

\section{Optimizing Cache Occupation by Array Padding}

We propose a new approach to determine how to pad arrays in order to optimize cache occupation, and consequently reduce miss rates. Padding techniques modify the portion of memory reserved for storing array data by including empty memory zones [1. We focus our analysis on intra-array padding, where the empty memory zones are included among the array dimensions that is, arrays are redimensioned by increasing the size in some of its dimensions.

\subsection{Single Loop Nest}

Consider first the case of a single loop nest and a single-level cache hierarchy. In our analysis we are specially interested in those arrays with a stride greater than the cache block size $(W)$ in the innermost loop of the nest. The cache occupation for these arrays may suffer from block replacements by self-interference, and some cache blocks may remain unoccupied. This happens when $\operatorname{Set} \operatorname{Stride}\left(X, I_{k}\right)>$ 1. This situation is very common in scientific applications, but the interesting problem occurs when the number of sets involved in the replacements is small. These cases shows an inefficient use of the cache space. Our goal is to change, in these cases, the array memory layout through padding to maximize the cache occupation, and consequently reduce the miss rate (due to self-replacements). Next lemma explains how to determine the dimension increments (intra-array padding) needed to maximize cache occupation.

Lemma. Given a loop $k$ in the nest and the array $X$, the occupation of the cache is maximized if SetStride $\left(X, I_{k}\right)$ and $C$ are mutually prime, that is, $G C D\left(\operatorname{Set} \operatorname{Stride}\left(X, I_{k}\right), C\right)=1$ (GCD condition).

Proof. We will prove that if $\operatorname{Set} \operatorname{Stride}\left(X, I_{k}\right)$ is relatively prime to $C$ then the first $C$ iterations of the loop $k$ touch different cache sets. In that case, if the loop $k$ is longer than $C$ then all cache sets are occupied by references to array $X$ in that loop. If it is shorter, the number of touched cache sets is maximum 


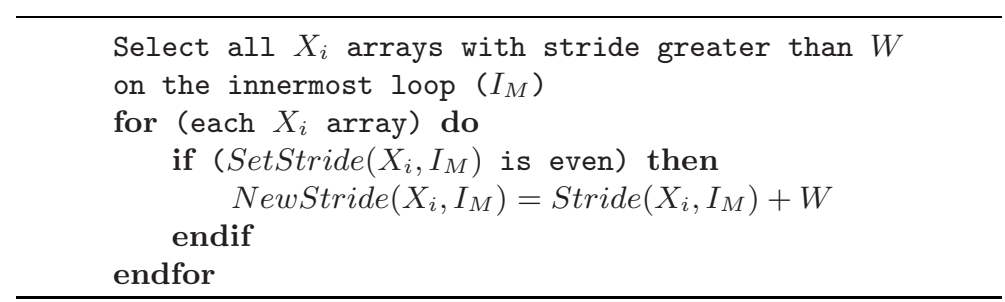

Fig. 1. Intra-array padding algorithm for a single loop nest and a single cache

(as many as the length of the loop). So, let us consider two of the first $C$ iterations of the loop $k$, say $p$ and $q$. Note that $0 \leq p, q<C$. Let us assume that references to $X$ in those two iterations are located to the same cache set, that is, $\operatorname{Set}\left(X, I_{k}^{p}\right)=\operatorname{Set}\left(X, I_{k}^{q}\right)$. In that case, from Eq. (11), we have, $\left(\operatorname{Set}\left(X, I_{k}^{L w}\right)+\right.$ $\left.p * \operatorname{Set} \operatorname{Stride}\left(X, I_{k}\right)\right) \bmod C=\left(\operatorname{Set}\left(X, I_{k}^{L w}\right)+q * \operatorname{Set} \operatorname{Stride}\left(X, I_{k}\right)\right) \bmod C$, or, eliminating the module operation, $\operatorname{Set}\left(X, I_{k}^{L w}\right)+p * \operatorname{Set} \operatorname{Stride}\left(X, I_{k}\right)=$ $\operatorname{Set}\left(X, I_{k}^{L w}\right)+q * \operatorname{Set} \operatorname{Stride}\left(X, I_{k}\right)+C * r$, where $r$ is an integer number. A bit of simplification gives, $(p-q) * \operatorname{SetStride}\left(X, I_{k}\right)=C * r$. Given that $\operatorname{Set} \operatorname{Stride}\left(X, I_{k}\right)$ and $C$ are mutually prime, $p-q$ must be divisible by $C$, as $r$ is an integer number. But $|p-q|$ is lower than $C$, so we came to a contradiction. Thus, references to $X$ in both iterations must touch different cache sets. And this must be true for any pair of such iterations.

Q.E.D.

In the case that $\operatorname{Set} \operatorname{Stride}\left(X, I_{k}\right)$ and $C$ are not mutually prime then the lemma does not hold. However, if $\beta$ is the greatest common factor between both values, then $\operatorname{Set} \operatorname{Stride}^{\prime}\left(X, I_{k}\right)=\operatorname{SetStride}\left(X, I_{k}\right) / \beta$ and $C^{\prime}=C / \beta$ are mutually prime. So, the lemma holds for these new reduced values. That means that the first $C^{\prime}$ iterations of loop $k$ touch different cache sets. The rest of them may cause set replacements. In addition, as $\operatorname{Set} \operatorname{Stride}\left(X, I_{k}\right)$ is assumed constant, the cache sets touched by these first iterations are $\beta$ sets apart. So, only the fraction $C / \beta$ cache sets are assured to be occupied.

The GCD condition can be used to define a simple algorithm (see Fig. 10 to compute the needed padding of an array in a nested loop for achieving maximum cache occupation. The key idea is touching the maximum number of cache sets when executing the iterations in the innermost loop. That permits to increase the cache set reuse by iterations of the next outer loop. In order to touch all the cache sets, we need to modify SetStride() to make it relatively prime to $C$. As $C$ is a power of two, an increment of SetStride() by one is enough. This is obtained by increasing BlockStride() by also one, or by increasing Stride() of the array by $W$ (remember that BlockStride() is assumed constant).

\subsection{Multiple Loop Nests (Global Code Optimization)}

In scientific codes it is very frequent that the same few arrays, usually referenced in the body of loop nests, are re-used during the whole program execution. To maximize the cache occupation for these array we may pad them satisfying the 


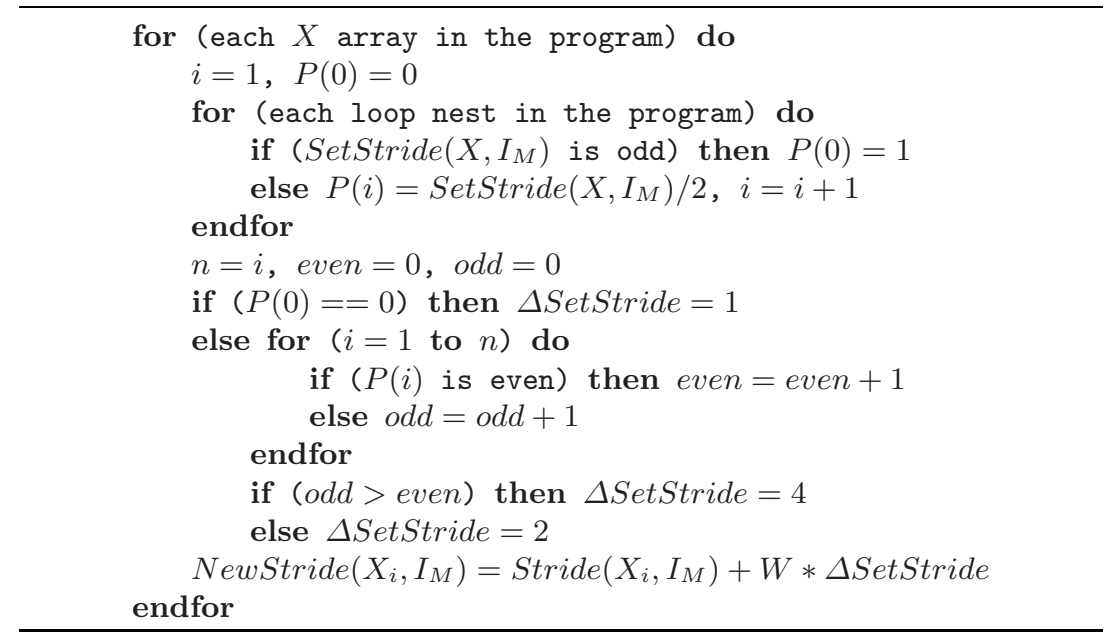

Fig. 2. Intra-array padding procedure for the whole program

GCD condition for all loop nests in the program that include such arrays. We have to bear in mind that we consider the arrays which are referenced over a non-contiguous dimension in the innermost loop of a nest. Then, for a specific array under consideration, we take all SetStride()'s corresponding to all nested loops, and calculate the stride increment (from now on, $\Delta$ Stride) needed to satisfy the GCD condition for every SetStride().

A new problem arises when for some loop nests SetStride() is even and for other loop nests SetStride() is odd. As $\Delta$ Stride must be the same for all loop nests, we need to calculate it so that it satisfies the GCD condition for all of them or, at least, it minimizes $G C D(\operatorname{Set} \operatorname{Stride}(), C)$, in order to maximize the number of the used cache sets.

In what follows we assume that the number of iterations of the different nested loops are similar. We also assume that the number of the odd and even values of SetStride()'s are similar. This is the worst case because we need to obtain a solution for the even values of SetStride()'s. In our approach, with a mixture of odd and even values for SetStride()'s, an even $\Delta$ Stride will be calculated, in order to not turning odd values into even ones. To minimize the number of the non touched cache sets, this even increment should minimize $G C D(\operatorname{Set} \operatorname{Stride}(), C)$. In addition, it is convenient that the increment being the smallest possible, in order to minimize the number of (empty) pad locations. Based on this idea, we have defined the algorithm shown in Fig. 2, that determines the array stride increment needed to maximize the occupation of the cache for the whole program. In the procedure a vector $P$ is computed containing the values $\operatorname{Set} \operatorname{Stride}() / 2$ for all even values of SetStride()'s. The first value of $P$ shows if there are odd values of SetStride()'s or not. With the even values, the minimum even increment in stride to minimize $G C D(\operatorname{Set} \operatorname{Stride}(), C)$ is computed for the whole program. 


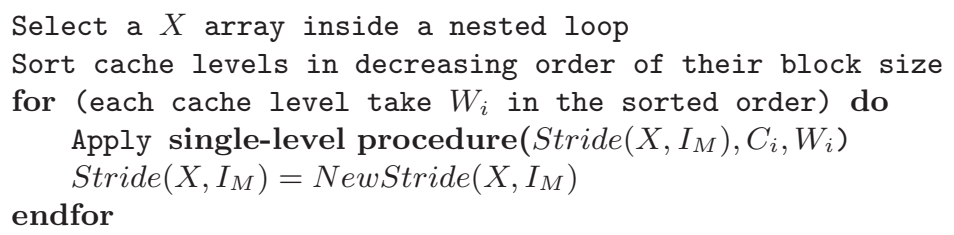

Fig. 3. Intra-array padding procedure for the complete cache hierarchy

\subsection{Complete Cache Hierarchy}

In the cache hierarchy the blocks may be the same size for all levels. In this case, the stride increment is the same for all cache levels, so we can apply the same algorithms described above. Otherwise, if block sizes are different over the cache hierarchy, we need to obtain a stride increment appropriate for each cache level.

The algorithm in Fig. 1 calculates the stride increment for a specific cache level. To pad the array for other cache level, we need to carry out the same algorithm changing SetStride() and the cache block size $(W)$. The method to accomplish this process starts with the cache level with the largest block size, continuing with the rest of levels in decreasing order of block size. As an example, consider a two-level cache hierarchy with different block sizes, $W 1$ and $W 2$, where $W 1<W 2$ (both are power of two). For this system, if Stride()/W2 is integer, then all the quotients, Stride ()$/ W 1,($ Stride ()$+W 2) / W 2),($ Stride ()$+$ $W 1) / W 1)$ and $($ Stride ()$+W 2+W 1) / W 1)$ are all also integer. However, the quotient $(\operatorname{Stride}()+W 2+W 1) / W 2)$ is non-integer. Despite this, its integer part satisfies the GCD condition. Based on these results, a simple algorithm has been developed to extend our padding approach to a cache hierarchy (see Fig. (3).

\section{Experimental Evaluation}

We first tested the basic padding method (single loop nest and one level cache) for a synthetic single loop nest example on a real machine, and for a small set of kernel benchmarks on a cache simulator. Second, we tested the full padding method (whole program optimization on a complete cache hierarchy) for a selection of benchmarks on a real machine. When using a real machine, different optimization levels where tested, obtaining similar results. In this paper we show those results for two optimization levels on two different processors.

\subsection{Basic Padding Method}

In this section we present the evaluation of the basic padding method for a simple test code, a double nested loop of $1000 \times 1000$ iterations $(i, j)$, where the loop body is $X(i, j)=3$, being $X$ a $1600 \times 1600$ single-precision floating-point array (4-byte words). The experiments were conducted in a MIPS R10K-processor system running in exclusive mode. This system has a 2-way set associative 32KByte L1 data cache with a 32 -Byte cache block $(C=512, L=2, W=8)$, 
Table 1. L1 (left) and L2 (right) data cache misses for different array paddings

\begin{tabular}{|c|c|c|c|c|c|c|c|c|r|}
\hline Arr. Dim. & SetStride & GCD & Exec. Time & L1 Misses & Arr. Dim. & SetStride & GCD & Exec. Time & L2 Misses \\
\hline \hline 1600 & 200 & 8 & 0.212 & $1,001,000$ & 1600 & 50 & 2 & 0.212 & 23,100 \\
\hline 1608 & 201 & $\mathbf{1}$ & 0.171 & 312,000 & 1632 & 51 & $\mathbf{1}$ & 0.214 & 19,500 \\
\hline 1616 & 202 & 2 & 0.214 & $1,001,000$ & 1664 & 52 & 4 & 0.219 & 27,640 \\
\hline 1624 & 203 & $\mathbf{1}$ & 0.169 & 310,000 & 1696 & 53 & $\mathbf{1}$ & 0.217 & 20,400 \\
\hline 1632 & 204 & 4 & 0.214 & $1,001,000$ & 1728 & 54 & 2 & 0.214 & 24,700 \\
\hline 1640 & 205 & $\mathbf{1}$ & 0.168 & 312,000 & 1760 & 55 & $\mathbf{1}$ & 0.212 & 18,600 \\
\hline 1648 & 206 & 2 & 0.216 & $1,001,000$ & 2048 & 64 & 64 & 0.548 & 698,500 \\
\hline 1664 & 208 & 16 & 0.217 & $1,001,000$ & 2176 & 68 & 4 & 0.217 & 22,200 \\
\hline
\end{tabular}

Table 2. Cache miss ratio for a $16 \mathrm{~KB}$ cache using a simulator

\begin{tabular}{|l|c|c|c|c|c|c|c|c|}
\hline & & & \multicolumn{7}{|c|}{ Cache Miss Ratio } \\
\hline Benchmark & Array Dim. & GCD & $\begin{array}{c}\mathrm{C}=1024 \\
\mathrm{~L}=1\end{array}$ & $\begin{array}{c}\mathrm{C}=512 \\
\mathrm{~L}=2\end{array}$ & $\begin{array}{c}\mathrm{C}=256 \\
\mathrm{~L}=4\end{array}$ & $\begin{array}{c}\mathrm{C}=128 \\
\mathrm{~L}=8\end{array}$ & $\begin{array}{c}\mathrm{C}=64 \\
\mathrm{~L}=16\end{array}$ & $\mathrm{C}=32$ \\
$\mathrm{~L}=32$
\end{tabular}

and a 2-way set associative 4-MByte L2 data cache with a 128-Byte cache block ( $C=16384, L=2, W=32$ ). The results correspond to codes compiled with the MIPSpro Fortran 90 (v. 7.30) compiler using the "O0" optimization option.

Considering the test code, we have $\operatorname{Stride}(X, i)=1$ and $\operatorname{Stride}(X, j)=1600$. For the L1 cache level we have, $\operatorname{BlockStride}(X, j)=1600 / 8=200$ (constant), $\operatorname{Set} \operatorname{Stride}(X, j)=200 \bmod 512=200$, and $\operatorname{GCD}(200,512)=8$. This result means that between two touched cache sets there are 7 other sets which are never used. With an increment by one of $\operatorname{SetStride}(X, j)$, we have $G C D(201,512)=1$ and $N$ ewStride $(X, j)=1600+8=1608$. Table 1 (left) shows the experimental results (execution time and L1 data cache misses) for different values of the second dimension of the array $X$. Besides, the table shows the $\operatorname{Set} \operatorname{Stride}(X, j)$ for each case and the values of $G C D(\operatorname{Set} \operatorname{Stride}(X, j), C)$. Note that the best results are obtained when the GCD condition holds, as expected.

For the L2 cache level, we have now BlockStride $(X, j)=1600 / 32=50$ (constant), SetStride $(X, j)=50 \bmod 16384=50$, and $G C D(50,16384)=2$. So, only half of the L2 cache is used. Table 1 (right) shows the experimental results for the L2 cache and different values for the array second dimension. The table shows three cases fulfilling the GCD condition. For these cases the minimum miss rate is obtained, as expected.

A different scenario corresponds to the evaluation of our basic padding method using a cache simulator, in order to test easily different cache configurations. The obtained results are shown in table 2, for three different benchmarks: test 
code (test code described above), liver (livermore kernel 3]), and mxm (matrix multiplication of square matrices). The first row in table 2 for each benchmark corresponds to the original dimension size of the array. The rest of rows include different padded dimension sizes. The smallest one (that is, the second row) corresponds to the increment given by our padding technique. For each cache configuration we measured the cache miss rate using our simulator. The results are given in the table as Cache Miss Ratio, that is obtained by dividing the cache miss rate for the original dimension size by the cache miss rate for the padded dimension size. This way, a value larger than 1 means that the miss rate was decreased after applying padding. In case of $m x m$, padding is not effective due to the small size of the data structures.

\subsection{Full Padding Method}

A different experimental setup was implemented testing a number of bechmarks from different suites (SPEC95 \& 2000, perfectB and NAS) on a Intel Pentium-4 platform. The computer system has a 8 -way set associative 16-KByte L1 data cache with a 64-Byte block size, and a 8-way set associative 1-MByte L2 data cache with a 64-Byte block size. The results shown here correspond to codes compiled with the Intel Fortran Compiler v. 9.0, using in this case the "O3" optimization level and "-align_none" option (in order to not interfere with padding). In addition, the code was executed in exclusive mode (hard real-time mode in the linux scheduler). Table 3 (top) shows the performance results applying our padding method for the complete cache hierarchy. Note that there are a significant performance improvement by padding the arrays.

In order to optimize the whole code, we first have to find all the nested loops where array references in a non contiguous dimension exist in the innermost loop. Next, we proceed by selecting one of these arrays. From this point on, SetStride()'s are calculated for every level of the cache hierarchy. For each one, the corresponding values of $G C D(\operatorname{Set} \operatorname{Stride}(), C)$ are also calculated, and from then, the stride increments. If the stride values are the same for all the loops, we only have to carry out the sum of the stride increments obtained for each level of the cache memory. Otherwise, we have to apply the corresponding padding algorithm. Table 3 (bottom) show the improvement obtained by applying our padding method to several benchmarks on the Pentium-4 machine.

\section{Related Work}

There is a great amount of work in the literature related to the design of compiler techniques for program locality exploitation. In [6] authors present an iterative method that uses ILP (Integer Linear Programming) to compute optimal solutions of memory layout transformations. Other works 78 also develop heuristic solutions to data layout and loop transformations, based on data reuse vectors.

Works based on Cache Miss Equations (CMEs) [5] are similar to our own proposal in some aspects but with different results. They analyze the cache 
Table 3. Improvement for a single loop nest (top) and the whole code (bottom) for the complete cache hierarchy in Pentium 4

\begin{tabular}{|c|c|c|c|c|c|c|}
\hline & Subroutine & $\begin{array}{c}\text { Original } \\
\text { array dim. }\end{array}$ & $\begin{array}{c}\text { Padded } \\
\text { array dim. }\end{array}$ & $\begin{array}{l}\% \text { Exec. time } \\
\text { improvement }\end{array}$ & $\begin{array}{c}\% \text { L1 num. misses } \\
\text { improvement }\end{array}$ & $\begin{array}{c}\% \text { L2 num. misses } \\
\text { improvement }\end{array}$ \\
\hline \multicolumn{7}{|l|}{$\begin{array}{l}\text { PerfectB } \\
\text { Bench. }\end{array}$} \\
\hline $\mathrm{adm}$ & hyd & $50,50,50$ & $56,52,50$ & 16.2 & 15.1 & 0 \\
\hline $\operatorname{arc} 2 d$ & scaldt & $500,500,4$ & $544,500,4$ & 11.3 & 15.7 & 13.6 \\
\hline dyfesm & mnlbyx & $500,500,3$ & $544,500,3$ & 7.1 & 1.2 & 44.4 \\
\hline flo52 & collc & $200,200,200$ & $200,205,200$ & 5.4 & 5.5 & 5.7 \\
\hline mg3d & march & 100,100 & 100,112 & 3.2 & 0.7 & 8.6 \\
\hline spec77 & horiz2 & 500,500 & 544,500 & 16.8 & 15.5 & 34.2 \\
\hline trfd & trfa & 500,500 & 544,500 & 18.3 & 3.8 & 0 \\
\hline \multicolumn{7}{|l|}{$\begin{array}{l}\text { NAS } \\
\text { Bench. }\end{array}$} \\
\hline appsp & spentax3 & $660,33,33$ & $660,35,34$ & 0 & 6.3 & 2.0 \\
\hline appbt & l2norm & $50,50,50,50$ & $56,52,56,50$ & 0 & 0.2 & 0 \\
\hline fftpde & transx & 1024,1024 & 1064,1024 & 120.0 & -1.7 & 90.0 \\
\hline \multicolumn{7}{|l|}{\begin{tabular}{|c|} 
SPEC CFP \\
Bench.
\end{tabular}} \\
\hline tomcatv & cal.res. & 513,513 & 540,520 & 588.2 & 211.5 & 103.8 \\
\hline applu & rhs & $20,50,50,100$ & $20,50,70,100$ & 5.7 & 4.7 & 2.9 \\
\hline swim & calc3z & 1335,1335 & 1352,1336 & 6.8 & 1.6 & 3.6 \\
\hline hydro2d & v.th.p & 1200,800 & 1224,800 & 433.2 & 907.4 & 173.3 \\
\hline mgrid & norm2u3 & $320,200,200$ & $344,201,200$ & 4.2 & 19.5 & 18.4 \\
\hline turb3d & wcal & $33,64,64$ & $34,68,64$ & 8.3 & -0.1 & 11.7 \\
\hline wave & RADFG & $100,100,100$ & $116,140,100$ & 5.4 & 5.5 & 5.7 \\
\hline tfft2 & $\operatorname{transc}$ & $500,30,500$ & $500,30,501$ & 6.5 & 11.7 & 0 \\
\hline
\end{tabular}

\begin{tabular}{|c|c|c|c|}
\hline & $\begin{array}{c}\text { \% Exec. time } \\
\text { improvement }\end{array}$ & $\begin{array}{c}\text { L1 num. misses } \\
\text { improvement }\end{array}$ & $\begin{array}{c}\text { L2 num. misses } \\
\text { improvement }\end{array}$ \\
\hline swim & 1.1 & 3.2 & -0.2 \\
\hline mgrid & 3.4 & 2.5 & 1.3 \\
\hline appsp & 4.8 & 2.8 & 3.7 \\
\hline fftpde & 1.2 & 3.9 & 10.4 \\
\hline
\end{tabular}

occupation through references to arrays in loops and define the algorithms to generate the CMEs and some optimizations, as padding. In [11] authors also use CMEs to propose a cost model that combined with a genetic algorithm carries out padding (and tiling) for a multi-level cache memory system.

The work in [2] is mainly focused on spatial locality optimization. The method provides a parameterized cost function based on polytopes and Ehrhart polynomials from the iteration space of a loop nest. We also use the polyhedron defined by the iteration space of a loop nest, but with a different objective. Our goal is to determine the cache occupation generated by this polyhedron. In [9] authors present a similar approach to ours for array padding. Their technique is iterative until no self-interference is caused. Our method, however, is a direct calculation, more accurate and with lower algorithmic complexity. Other work [10] computes the conflict distance of array references by directly linearizing the uniformly-generated references. In [12] authors analyze iterative stencil loops and use array padding to remove conflict misses after tiling the loops.

Finally, the work in [4] shares with our approach a similar treatment of the cache and the program code. However, we also take into account how array 
data is stored in memory, and we introduce the new notions of cache block and set strides and work with them to develop the padding algorithms. These facts, to some extent, complete their work, justifying some of the results they obtain.

\section{Conclusions}

We presented a parameter-based cache model that is used as a framework to determine how to pad arrays in order to optimize program locality. In fact, the model permits to maximize cache occupation when arrays are reference within loop nests. This model is used to develop array padding algorithms in different scenarios: single loop nest and multiple loops, single and multiple cache levels. We showed that simple padding techniques are very useful to obtain respectable performance improvements for a variety of scientific codes.

\section{References}

1. Bacon, D.F., Graham, S.L., Sharp, O.J.: Compiler Transformations for HighPerformance Computing. ACM Computing Surveys 26(4), 345-420 (1994)

2. Clauss, P., Meister, B.: Automatic Memory Layout Transformation to Optimize Spatial Locality in Parameterized Loop Nests. ACM Computer Architecture News 28(1), 11-19 (2000)

3. Coleman, S., Mckinley, K.S.: Tile Size Selection Using Cache Organization and Data Layout. In: ACM Conf. on PLDI. La Jolla (CA), pp. 279-290 (1995)

4. Ferrante, J., Sarkar, V., Thrash, W.: On Estimating and Enhancing Cache Effectivenes. Work. on Languages and Compilers for Parallel Computers (1991)

5. Ghosh, S., Martonosi, M., Malik, S.: Cache Miss Equations: A Compiler Framework for Analyzing and Tunning Memory Behaviour. ACM TOPLAS 21(4), 703-746 (1999)

6. Kandemir, M., Banerjee, P., Choudhary, A., Ramanujam, J., Ayguade, E.: An Integer Linear Programming Approach for Optimizing Cache Locality. In: ACM Int'l. Conf. on Supercomputing Rhodes, pp. 500-509 (1999)

7. Kandemir, M., Choudhary, A., Ramanujam, J., Banerjee, P.: Improving Locality Using Loop and Data Transformations in an Integrated Framework. In: ACM/IEEE Int'l. Symp. on Microarchitecture. Dallas (TX), pp. 285-297 (1998)

8. O'Boyle, M., Knijnenburg, P.: Integrating Loop and Data Transformations for Global Optimizations. In: IEEE Int'l. Conf. on Parallel Architectures and Compilation Techniques., Paris, pp. 12-19 (1998)

9. Panda, P., Nakamura, H., Dutt, N., Nicolau, A.: A Data Alignment Technique for Improving Cache Performance. In: Int'l. Conf. on Computer Design: VLSI in Computers and Processors., Austin (TX), pp. 587-592 (1997)

10. Rivera, G., Tseng, C.W.: Data Transformations for Eliminating Conflict Misses. In: ACM Conf. on PLDI, Montreal, pp. 38-49 (1998)

11. Vera, X., Abella, J., Llosa, J., González, A.: An Accurate Cost Model for Guiding Data Locality Transformations. ACM TOPLAS 27(5), 946-987 (2005)

12. Li, Z., Song, Y.: Automatic Tiling of Iterative Stencil Loops. ACM TOPLAS 26(6), 975-1028 (2004) 